\title{
A Novel Magnetic Levitation Haptic Device for Augmentation of Tissue Stiffness Perception
}

\author{
Qianqian Tong, Zhiyong Yuan, Mianlun Zheng, Weixu Zhu, Guian Zhang, Xiangyun Liao \\ School of Computer, Wuhan University, Hubei, China
}

\begin{abstract}
In medical training especially in palpation surgery, it is important for surgeons to perceive tissue stiffness. We design a novel magnetic levitation haptic device based on electromagnetic principles to enhance the perception of tissue stiffness in a virtual environment. The user can directly sense virtual tissues by moving a magnetic stylus in the magnetic field generated by the coil array of our device. To fully use the effective magnetic field, we devise an adjustable coil array and provide a reasonable explanation for such design. Moreover, we design a control interface circuit and present a self-adaptive fuzzy proportion integration differentiation (PID) algorithm to precisely control the coil current. The quantitative experiment shows that the experimental and simulation data of our device are consistent and the proposed control algorithm contributes to increasing the accuracy of tissue stiffness perception. In qualitative experiment, we recruit 22 participants to distinguish tissues of different stiffness and detect tissue abnormality. The experimental results demonstrate that our magnetic levitation haptic device can provide accurate perception of tissue stiffness.
\end{abstract}

Keywords: magnetic levitation haptic device, stiffness perception augmentation, adjustable coil array, self-adaptive fuzzy PID

\section{Concepts: $\bullet$ Hardware $\rightarrow$ Haptic devices;}

\section{Introduction}

In open surgery, surgeons usually detect tissue abnormality by manually palpating the biological tissue. However, operators cannot make direct contact with soft tissues in virtual reality (VR) applications such as surgical simulation, which makes it difficult to detect tissue abnormality[Gwilliam et al. 2010]. In this medical task of detecting tissue abnormality, surgeons usually perceive tissue stiffness and diagnose the areas those are stiffer than surrounding tissues as possible tumors [De Gersem et al. 2005]. There are mainly two kinds of methods to display stiffness information: pseudo-haptic feedback and haptic feedback. Pseudo-haptic feedback is based on tissue stiffness maps provided by rolling mechanical imaging [ $\mathrm{Li}$ u et al. 2008; Liu et al. 2010; Liu et al. 2011; Li et al. 2012] and does not require real haptic devices. The pseudo-haptic feedback is low-cost, but it cannot precisely simulate the human's way of perceiving information like real haptic devices. Stetten et al. [Stetten et al. 2011] designed a haptic augmented reality (AR) system, and the experimental results showed that the force augmentation induced by the device was well perceived by participants. Therefore,

*e-mail:zhiyongyuan@whu.edu.cn

Permission to make digital or hard copies of all or part of this work for personal or classroom use is granted without fee provided that copies are not made or distributed for profit or commercial advantage and that copies bear this notice and the full citation on the first page. Copyrights for components of this work owned by others than ACM must be honored. Abstracting with credit is permitted. To copy otherwise, or republish, to post on servers or to redistribute to lists, requires prior specific permission and/or a fee. Request permissions from permissions@acm.org. (c) 2016 ACM.

VRST '16,, November 02-04, 2016, Garching bei München, Germany

ISBN: 978-1-4503-4491-3/16/11 ..\$15.00

DOI: http://dx.doi.org/10.1145/2993369.2993377

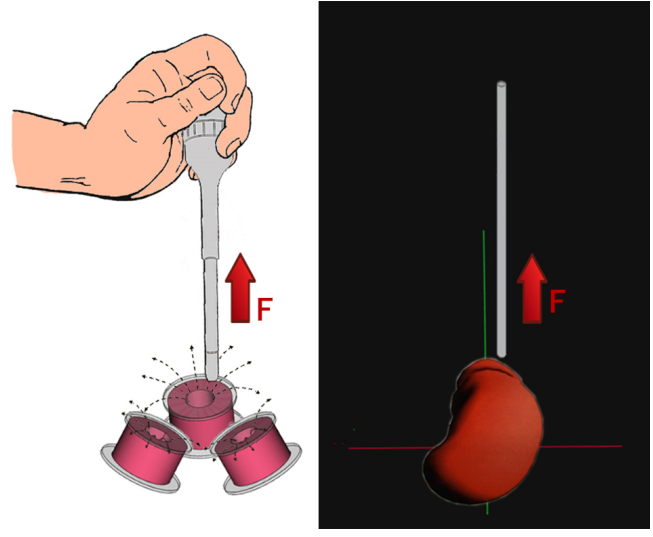

Figure 1: Principle of the designed haptic device for enhancing the perception of tissue stiffness.

users prefer to use haptic devices to enhance the sensing of tissue stiffness, and the devices help them to detect and position abnormal tissues in surgical simulation or other practical applications such as robot-assisted minimally invasive surgery (RMIS), which has been widely applied in recent years [Li et al. 2014].

There have been numerous studies on the augmentation of tissue stiffness perception using haptic devices such as the most widely used Phantom. Nisky et al. [Nisky et al. 2012] combined a mechanical simulator with Phantom to perform the perception of stiffness in laparoscopy. Son et al. [Son et al. 2012] applied Phantom as the master device to perceive soft tissues in teleoperation experiments. However, Phantom suffers inherent mechanical friction and the limited movement of its stylus. Wu et al. [Wu et al. 2011] adopted a magnetic levitation haptic interface (named MLHD) in perceptual discrimination. Though MLHD has no mechanical friction, its movement is also limited. In addition, air-jet and ultrasonic radiation pressure are used to simulate haptic perception [Arafsha et al. 2015] : The AIREAL device designed by Disney Research [Sodhi et al. 2013] is an example of the air-jet tactile feedback which can be used with Kinect in entertainment based applications; Hoshi et al. [Hoshi et al. 2009] developed an interactive holographic system with tactile feedback by combining 4 ultrasound transducer arrays and Long et al. [Long et al. 2014] proposed a method for creating three-dimensional haptic shapes in mid-air using focused ultrasound. Nevertheless, there are some disadvantages of using the above two contactless methods to produce haptic feedback [Arafsha et al. 2015]: The air-jet method lacks spatial and temporal qualities that are necessary for multimedia applications due to the physical properties of air [Hoshi 2011]; The short travelling distance, unwanted noise, and safety issues of ultrasonic method limit its performance for haptic applications.

In this paper, we design a magnetic levitation haptic device for the augmentation of tissue stiffness perception in a virtual environment. As shown in Figure 1, the device is based on electromagnetic principles and current-carrying coils generate a magnetic field in space, which is the operation space of our device. When interacting with the soft tissue in a virtual environment, users feel the same force 
as the virtual stylus in the operation space by using a magnetic stylus (a stylus equipped with a small magnet, and the material of the magnet is NdFe52). Therefore, the user experiences the augmented perception of tissue stiffness in a natural manner. We simulate the effective magnetic field (EMF) generated around current-carrying coils and find that the magnetic field can be sufficiently used when the coils on an appropriate attitude. We design an adjustable coil array to make it convenient for adjusting the coil attitude. In addition, we design a control interface circuit to drive the coils based on ARM Cortex-M Microcontroller Unit (MCU) and H-bridge and adopt a self-adaptive fuzzy proportion integration differentiation (PID) algorithm to make the coil current more stable and precise. Thus we tackle the problem of unstable coil current caused by the reality that the coil current decreases as the coil becomes heated during the period of using the haptic device so as to improve the precision of the perception.

The rest of this paper is organized as follows. Section 2 presents a detailed review of related work. Section 3 describes the design of our magnetic levitation haptic device. Section 4 describes the experiments and results. Section 5 concludes with a discussion of the results and conclusions are drawn in Section 6.

\section{Related work}

\subsection{Tissue stiffness perception}

Many researchers have studied the augmentation of tissue stiffness perception. Bethea et al. [Bethea et al. 2004] evaluated a visual force feedback system developed for the da Vinci Surgical System, and the force at the tip of the surgical instrument was represented by a color bar. Horeman et al. [Horeman et al. 2012] augmented the display of their laparoscopic training platform with an arrow that continuously informed the trainee about the magnitude and direction of the applied force. Li et al. [ $\mathrm{Li}$ et al. 2012] proposed a tissue stiffness simulation technique based on tissue stiffness maps provided by rolling mechanical imaging using pseudo-haptic feedback.

The above studies do not directly assess the perception of stiffness. As mentioned above, haptic devices can simulate the ways humans perceive information more closely thus to enhance the perception of tissue stiffness. Song et al. [Song et al. 2005] designed a stiffness display interface device that was consisted of a thin elastic beam and an actuator to adjust the length of the beam, and the human fingertip felt as if directly touch the virtual objects by using the device. Kesner et al. [Kesner and Howe 2011] developed a motion-compensated actuated catheter system that enabled users to achieve more tactile information by providing haptic feedback during palpation procedures. $\mathrm{Li}$ et al. [Li et al. 2014] created a virtual tissue model and users can identify tumors embedded in the tissue model using a Phantom device. In addition, Quek et al. [Quek et al. 2014] designed a 1 degree of freedom (DOF) skin stretch device to augment stiffness perception.

The above haptic devices used for enhancing stiffness perception are mechanical. However, mechanical devices possess inheren$\mathrm{t}$ friction, which can affect the haptic experience. The MLHD [Robotics 1993; Berkelman and Hollis 2000] uses Lorentz forces for actuation, there are no motors, gears or bearings, as well as the device is free of static friction and its mechanical responses is fast. $\mathrm{Wu}$ et al. [Wu et al. 2011] used the MLHD device for perceptual discrimination. But the operation space of the MLHD is limited result from the limited movement of its stylus. We aim to design a novel magnetic levitation haptic device with an untethered magnetic stylus and higher accuracy for the augmentation of tissue stiffness perception.

\subsection{Magnetic levitation devices}

Hollis initially used Lorentz magnetic levitation to design a haptic feedback system called Magic Wrist [Robotics 1993; Hollis et al. 1991]. It holds up a six-angle suspension via Lorentz force and optical sensors, and the outputs of force and torque control the 6DOF movement of the suspension [Hollis Jr and Salcudean 1992]. Berkelman et al. winded coils around the handle as a suspension. The coils cut magnetic induction lines generated by pairs of permanent magnets to realize haptic feedback when the suspension handle moves or rotates [Berkelman and Hollis 2000; Berkelman and Hollis 1997; Berkelman 2007]. Given the limitation of translational and rotational range of the suspension handle, Berkelman et al. extended the motion range by designing a planar array which contains 10 cylindrical electromagnetism coils, providing force feedback for the suspense stylus on the planar array [Berkelman and Dzadovsky 2009]. Subsequently, they increased the number of coils to 27. That novel solution provides 6-DOF feedback force [Berkelman et al. 2012; Berkelman et al. 2013]. Hu et al. [Hu 2005] invented a type of electromagnetism based haptic feedback system. Then they designed an open surgery simulation and training system, and completed a preliminary experiment using a 1-DOF palpation simulation.

Researches of existing magnetic haptic devices have not yet provided a reasonable explanation of the methods for placing the coils (that is, how to determine the position and angle of coils). Berkelman simply placed coils on a plane in a hexagon [Berkelman and Dzadovsky 2008; Berkelman and Dzadovsky 2013]. Hu decentralized the coils into a concave area [Hu 2005; Hu et al. 2006]. We resolve the problem above by simulating and analyzing the EMF area generated by the coils of different positions and angles based on the finite element method (FEM). In addition, users cannot perceive tissue stiffness accurately if the current of the coils cannot be precisely controlled, which may affect the detection of tissue abnormality. In this paper, we design a control interface circuit to drive the coils and adopt a self-adaptive fuzzy PID algorithm to precisely adjust the coil current.

\section{Materials and Methods}

\subsection{Overall design of our haptic device}

Our magnetic levitation haptic device is based on the principle of electromagnetics. Current-carrying coils generate a magnetic field in operation space. When the magnetic stylus is fixed at a certain position in the magnetic field, the user senses a stable force feedback if the coil current is constant. Moreover, the user will feel changeable force feedback when the coil current or the position of the magnetic stylus changes. The designed haptic device can provide users with smooth forces by changing the coil current and has no mechanical friction in the process of interaction.

As shown in Figure 2, our magnetic levitation haptic device is composed of stereo vision tracking module, coil array, magnetic stylus, coil driver module and virtual scene display module, etc. When the operator uses the magnetic stylus to interact with objects in the virtual environment, the stereo vision tracking module tracks the position information of the magnetic stylus in real time and sends it to the virtual scene display module which computes the feedback force according to the previously established interaction model. Then, we adopt the method of [Berkelman and Dzadovsky 2013] to calculate the current for each coil. The driver module intelligently adjusts the current of each coil, thus making the coil array generate the magnetic field corresponding to the interactive process. Finally, the magnetic stylus receives the same force as the interactive process and transmits it to the user thus he can distinguish 
different objects according to the feedback force. The movement of the magnetic stylus is flexible, thus the whole interaction closely resembles the natural manner.

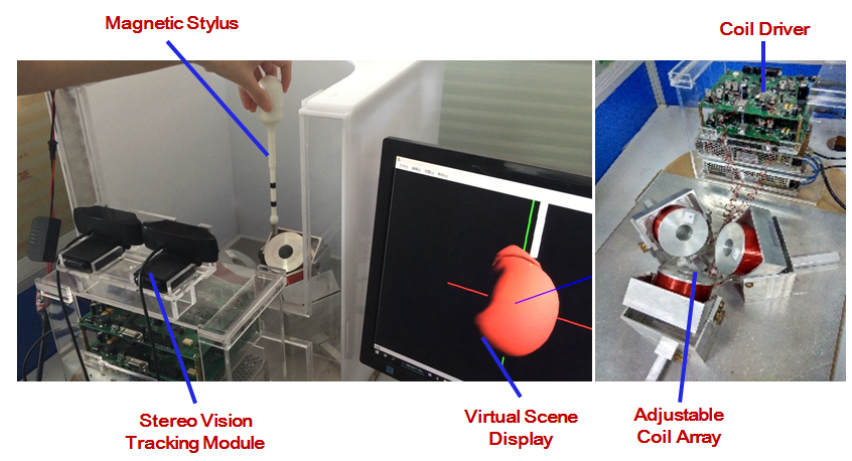

Figure 2: Overall design of our magnetic levitation haptic device.

We use binocular vision to navigate the interaction process thanks to its advantages of no contact, simple operation and good stability, etc. Markers (the black parts on the magnetic stylus shown in Figure 2) are set on the magnetic stylus, which are used to indicate the magnetic stylus's position. As a result, the binocular vision module needs to merely track the markers to locate the magnetic stylus, which is helpful to avoid dealing with redundant information, and we realize real-time tracking and positioning of the magnetic stylus by adopting this method.

\subsection{Coil configuration}

Our magnetic levitation haptic device uses three current-carrying cylindrical coils to produce EMF. Each cylindrical coil is consist of many toroidal coils. The whole cylindrical coil is considered as a superposition of multiple uniformly distributed toroidal coils. The parameters of each cylindrical coil are as follows: axial length $\mathrm{H}=62 \mathrm{~mm}$; outer radius of coils $\mathrm{R} 2=23 \mathrm{~mm}$; inner radius $\mathrm{R} 1=13$ $\mathrm{mm}$; number of coil turns $\mathrm{N}=1041$; and the coil material is copper.

As described in section 2, Berkelman placed the coils on a plane in a hexagon [Berkelman and Dzadovsky 2008; Berkelman and Dzadovsky 2013]. Hu decentralized the coils into a concave area [Hu 2005; Hu et al. 2006]. To reasonably design the coil array, we simulated the characteristic of the magnetic field generated by a current-carrying coil using the Ansoft Finite Element Analysis and Matlab. For a single coil, the electromagnetic force exerted on the magnetic stylus decreases as it leaves the coil array. In addition, the literature [Berkelman and Dzadovsky 2013] showed that the equation used for solving each coil current may have no theoretical solution, so the magnetic stylus cannot provide the needed feedback force to the user in such a case. Therefore, a key factor to design a haptic device for the augmentation of tissue stiffness perception is the EMF, namely, a magnetic field area where the current of each coil to be loaded can be solved.

We simulate and analyze the relationship between the EMF and the coil attitude based on FEM. As shown in Figure 3, the red part denotes the attitude of the three coils; the blue part shows a sphere, and the EMF area is in the interior of the sphere. The top figures show the relationship between the EMF and the coil angle varies from $0^{\circ}$ and $90^{\circ}$. We can see that the EMF gradually expands with an increasing angle, whereas its position is far from the coil plane. The bottom shows the relationship between the EMF and the distances between three coils. Similarly, the EMF area increases with an increasing distance, and the EMF is also increasingly far from

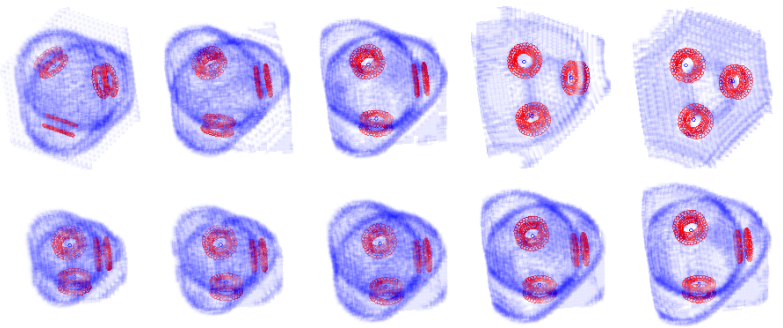

Figure 3: The relationship between the EMF and coil attitude. The top figures show the relationship between the EMF and coil angle; the bottom show the relationship between the EMF and the distance among the three coils.

three coils. As mentioned above, the electromagnetic force will decrease when the magnetic stylus is far from the coil plane; so when the EMF is far from the coil plane, the range of feedback force generated by our haptic device will be affected.

We now know that the coil attitude will affect the performance of our haptic device from the simulation analysis. In order to adjust the coil attitude conveniently, we design a special configuration of the mechanical part. As is shown in Figure 4, we design a pedestal which can be used to adjust the attitude of the three coils, and it contains three sliding supports, three coil containers and three coils. The sliding rails are placed on the pedestal, the sliding supports are placed $120^{\circ}$ apart on the sliding rails, the coil containers are placed on the sliding supports, and the coils are placed on the containers. Three coils can be freely adjusted on the plane and are parallel to the sliding rails and the axle rolls are used to adjust the angle. By adjusting the coil attitude, we can choose a better coil attitude for different objects in a virtual environment by perceiving the tissue stiffness.

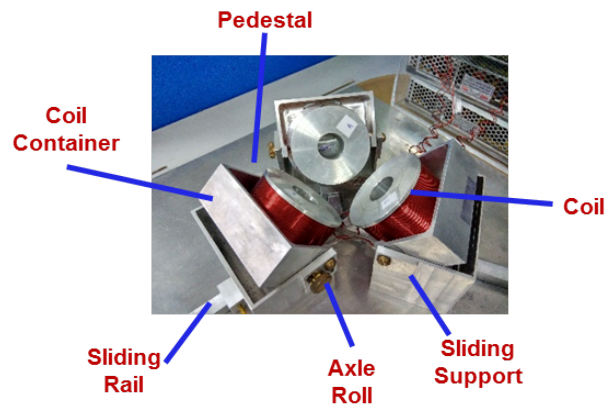

Figure 4: Coil array consist of three coils. The coil attitude can be freely adjusted.

\subsection{Coil driver circuit}

We design a coil driver circuit to control the coil current. As shown in Figure 5, the ARM Cortex-M MCU is the core controller which generates a pulse width modulation (PWM) signal to drive the $\mathrm{H}$ bridge circuit. The $\mathrm{H}$ bridge circuit adopts the PWM signal to control the state (conduction or cutoff) of the metal-oxidesemiconductor field-effect transistors (MOSFETs) on the arms of the $\mathrm{H}$ bridge circuit to achieve an variation in the current direction of coils.

We choose the complementary PWM driving mode to control the coil current. As shown in Figure 5, Q1, Q2, Q3 and Q4 are all 
NMOSFETs (NMOSs). Q1 and Q2 compose a group of NMOSs alternately conducting on the same side of the H-bridge, and Q3 and Q4 are another group. If Q1 and Q4 conduct at the same time, the two sides A and B of the coil would have forward voltage. This would be backward voltage when Q2 and Q3 conduct. Adjusting the duty ratio of the two PWM signals can control the average current in the coil. If the duty ratio of the PWM signals increases, the coil current will increase, and the haptic device will thereby provide a larger force feedback

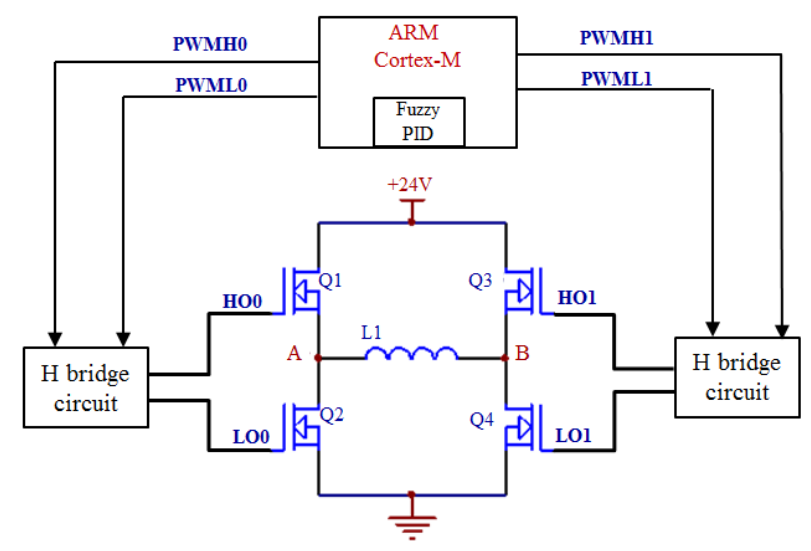

Figure 5: The coil driver circuit to control the coil current and complementary PWM driving mode.

We use two H-bridge driver chips to realize full-bridge driving. The PWM signal generated by the ARM Cortex-M MCU first passes an opt coupler to ensure that the MCU can work well. For a halfbridge, the PWM signals of two channels are send to an AND gate chip, and its output is used to is protect the NMOSs because it will be at a high level when the two input signals are both at high levels, thus can lock the input channels of the H-bridge driving chip.

\subsection{Coil current control}

Because of the influence of external environment, electromagnetic interference, etc., there would be a certain deviation between the actual coil current and the needed current in an interactive process. As the conduction time increases, the coil resistance will increase, resulting in feedback force diminishing and the user feeling the tissue as softer. Here we adopt a self-adaptive fuzzy PID algorithm to resolve this problem.

The actual current should be acquired to achieve the precise control of the current. Because we use the PWM signal to drive the coils, the actual current of the coil cannot be directly acquired. In this paper, we use the current sensor to detect the voltage across the sampling resistor, which is in series with the coil. The current value is linearly transformed into a PWM signal. Then, the ARM Cortex-M MCU measures the duty ratio of the signal by its timer and calculates the actual current of the coil.

The difficulty of the classical PID algorithm lies in the problem of parameter self-tuning in the proportional, integral and differential link. $K_{p}, K_{i}$, and $K_{d}$ are respectively proportional, integral and differential coefficients of PID algorithm. For general accuracy requirements, the patchwork approach can be used. To improve the performance of our magnetic levitation haptic device, and ensure the accurate stiffness perception of the virtual objects, we adopt the idea of fuzzy reasoning. The on-line self-tuning of $K_{p}, K_{i}$, and $K_{d}$ are performed according to different current deviations $e(k)$ and variation rates $e_{c}(k)$.
The process of the self-adaptive fuzzy PID algorithm to realize precise control of the coil current is shown in Figure 6. We adopt the incremental PID algorithm, supposing that $\Delta k_{p}, \Delta k_{i}$, and $\Delta k_{d}$ are the correction values of $K_{p}, K_{i}$, and $K_{d}$, respectively. Firstly, the deviation $e$, deviation variation rate $e_{c}$ and the outputs $\Delta k_{p}, \Delta k_{i}$, and $\Delta k_{d}$ are fuzzed using a triangular membership function, denoting the fuzzy linguistic variables of $e, e_{c}$ as $E, E_{c}$. The fuzzy output is then calculated by solving the fuzzy relational equation from a fuzzy reasoning that uses the smaller value according to the designed fuzzy rule table. Then, the precise value is calculated using the weighted average method to acquire the actual outputs of $\Delta k_{p}, \Delta k_{i}$, and $\Delta k_{d}$ after the scale transformation. Finally, we achieve the PID parameters $K_{p}, K_{i}$, and $K_{d}$, which are used to calculate the incremental value of the PWM signal. Therefore, the ARM Cortex-M MCU accomplishes control of the coil current in real time.

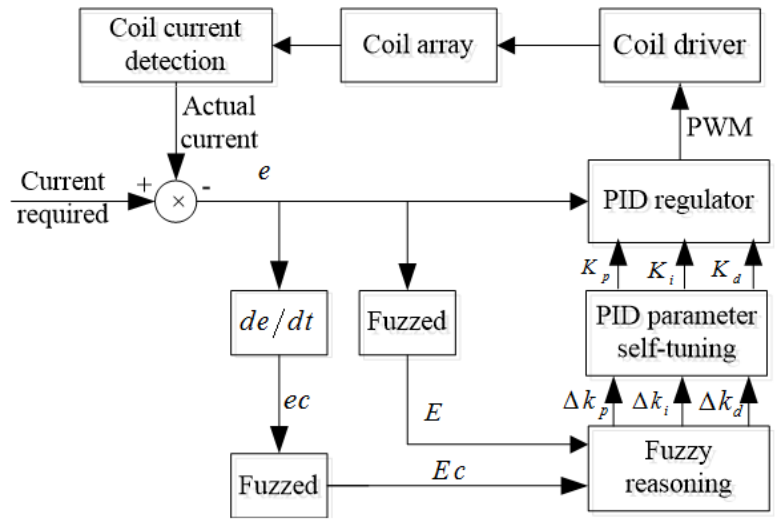

Figure 6: Flowchart of the self-adaptive fuzzy PID algorithm to realize the accurate adjustment of the coil current.

In summary, Algorithm 1 describes the algorithm of the coil intelligent current control of our magnetic levitation haptic feedback device.

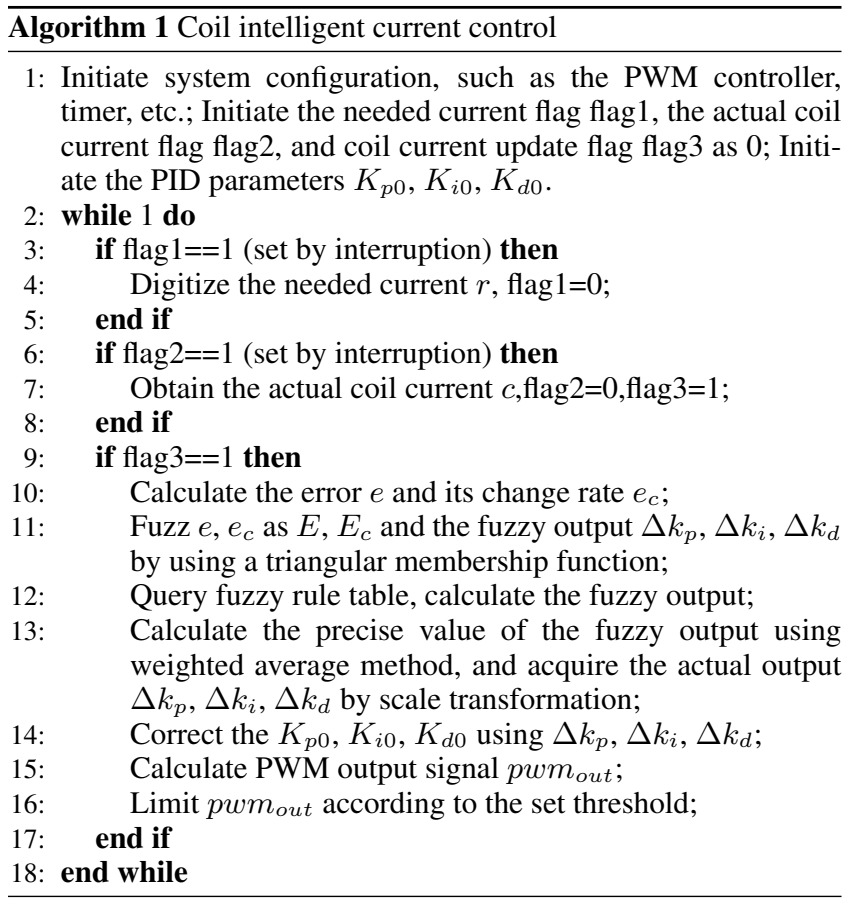


Table 1: Comparison between the experimental and simulation data of magnetic induction intensity for a single coil.

\begin{tabular}{|c|c|c|c|c|c|c|c|c|}
\hline \multirow{2}{*}{$\begin{array}{c}\text { Distance } \\
(\mathbf{m m})\end{array}$} & \multicolumn{9}{|c|}{ Experimental data $(\mathbf{m T})$} & \multicolumn{4}{|c|}{ Simulation data $(\mathbf{m T})$} \\
\cline { 2 - 9 } & 0.5 & 1.0 & 1.5 & 2.0 & 0.5 & 1.0 & 1.5 & 2.0 \\
\hline 69 & 15 & 28.8 & 40 & 53.2 & 13 & 26.3 & 39 & 52.1 \\
\hline 74 & 9.4 & 18 & 26.6 & 35.6 & 8.26 & 16.6 & 25 & 32.7 \\
\hline 79 & 6.1 & 11.8 & 17.5 & 21.2 & 5.47 & 11.1 & 16 & 22.1 \\
\hline 84 & 4.1 & 7.8 & 11.9 & 14.2 & 3.64 & 7.56 & 11 & 14.4 \\
\hline 89 & 2.6 & 5.1 & 7.7 & 9.9 & 2.7 & 5.3 & 8.0 & 10.8 \\
\hline 94 & 2.0 & 4.0 & 5.8 & 7.1 & 2.03 & 4.07 & 6.0 & 7.96 \\
\hline 99 & 1.5 & 2.9 & 4.4 & 5.3 & 1.54 & 3.12 & 4.6 & 6.18 \\
\hline 104 & 1.1 & 2.2 & 3.3 & 3.9 & 1.14 & 2.29 & 3.5 & 4.67 \\
\hline 109 & 0.9 & 1.7 & 2.4 & 3.1 & 0.99 & 1.20 & 3.0 & 3.99 \\
\hline 114 & 0.7 & 1.4 & 1.9 & 2.5 & 0.81 & 1.65 & 2.5 & 3.28 \\
\hline 119 & 0.5 & 1.1 & 1.6 & 1.9 & 0.69 & 1.38 & 2.1 & 2.74 \\
\hline 124 & 0.4 & 0.9 & 1.4 & 1.7 & 0.56 & 1.12 & 1.67 & 2.28 \\
\hline
\end{tabular}

\section{Experiments}

To evaluate the performance of our magnetic levitation haptic device, we conducted quantitative and qualitative experiments. In the quantitative experiment, we compare the experimental and simulation data of magnetic induction intensity generated by a single coil as well as three-coil, and performance of self-adaptive fuzzy PID algorithm and the classical PID algorithm were compared. In the qualitative experiment, the designed device was subjectively compared with the Phantom Omni device by recruiting participants to distinguish objects of different stiffness, detect tissue abnormality and complete a questionnaire.

\subsection{Quantitative experiment}

We adopted Ansoft software to simulate the magnetic field generated by a single coil and three-coil. The distribution of electromagnetic force exerted on a small magnet at 2A current are shown in Figure 7. The colors indicate the magnitude of electromagnetic force and red is stronger while blue is weaker. Figure 7 (a) shows the distribution of electromagnetic force at the plane $\mathrm{z}=2 \mathrm{~mm}$ (the range of $x, y$ is $[-35 \mathrm{~mm}, 35 \mathrm{~mm}]$ ) of single-coil magnetic field. When $\mathrm{z}$ is identical, the force gets stronger as the small magnet approaches the center axis of the coil until reaches the peak. Figure 7 (b) shows the force distribution at the plane $\mathrm{z}=65 \mathrm{~mm}$ (the range of $x, y$ is $[-70 \mathrm{~mm}, 70 \mathrm{~mm}]$ ) of three-coil. We can see that the electromagnetic force also gets stronger when the small magnet approaches the coils, while it gets weaker in the clearances of the three coils.

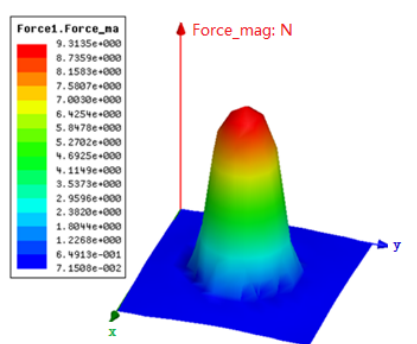

(a)

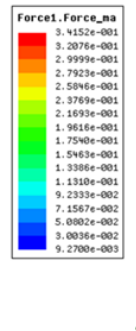

(b)
Figure 7: The distribution of electromagnetic force exerted on a small magnet by a single coil and three-coil.

\subsubsection{Magnetic induction intensity of a single coil}

We performed two groups of comparative experiments for the experimental and simulation data. One is the relationship between the magnetic induction intensity and the position when the coil current is steady. The other is the relationship between the magnetic field distribution and the current. In the circumstance with the coil currents of $0.5 \mathrm{~A}, 1 \mathrm{~A}, 1.5 \mathrm{~A}$ and $2 \mathrm{~A}$, the magnetic field of a certain continuous position of the $\mathrm{z}$ axis was measured from $(0,0,69)$ to $(0,0,124)$ with a step size of $5 \mathrm{~mm}$. Table 1 shows a comparison of the experimental and simulation data. As shown in Figure 8, the horizontal coordinate represents the position of the central axis, and the vertical coordinate represents the magnetic induction intensity in space. It is apparent that the simulation data and the experimental data are significantly consistent. The curve of Figure 8 describes that the magnetic induction intensity of the single coil first significantly decreases and then slowly after a certain critical value with an increasing distance. In the same position, the magnetic induction intensity also doubles when the coil current doubles.

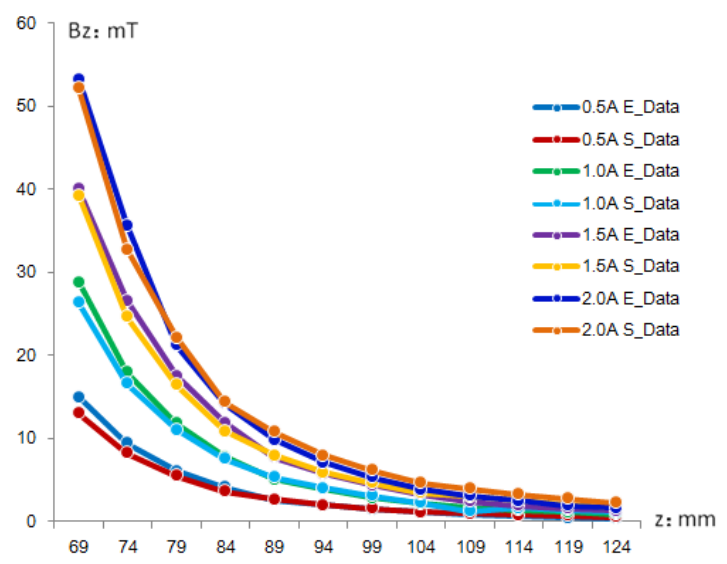

Figure 8: Comparison chart between the experimental and simulation data of magnetic induction intensity for a single coil.

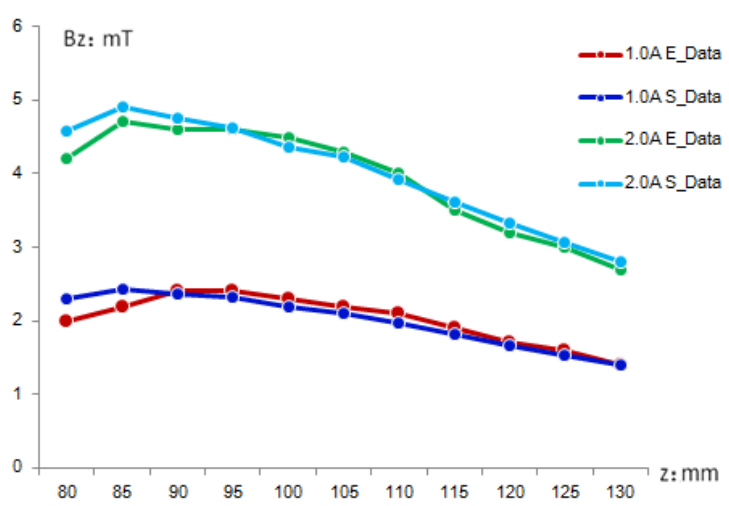

Figure 9: Comparison chart between the experimental and simulation data of magnetic induction intensity for three-coil.

\subsubsection{Magnetic induction intensity of three-coil}

In this section, the magnetic induction intensity of three-coil is measured at $1 \mathrm{~A}$ and $2 \mathrm{~A}$ in different positions on the coil center axis (from $(0,0,80)$ to $(0,0,130)$ with the step of $5 \mathrm{~mm})$. Table 2 shows the experimental and simulation data of magnetic induction intensity on the coil center axis, and the three coils are placed according 
Table 2: Comparison between the experimental and simulation data of magnetic induction intensity for a single coil.

\begin{tabular}{|c|c|c|c|c|}
\hline \multirow{2}{*}{$\begin{array}{c}\text { Distance } \\
(\mathbf{m m})\end{array}$} & \multicolumn{2}{|c|}{ Experimental data $(\mathbf{m T})$} & \multicolumn{2}{c|}{ Simulation data $(\mathbf{m T})$} \\
\cline { 2 - 5 } & 1.0 & 2.0 & 1.0 & 2.0 \\
\hline 80 & 2.29 & 4.57 & 2 & 4.2 \\
\hline 85 & 2.44 & 4.9 & 22 & 4.7 \\
\hline 90 & 2.36 & 4.75 & 2.4 & 4.6 \\
\hline 95 & 2.33 & 4.63 & 2.4 & 4.6 \\
\hline 100 & 2.19 & 4.37 & 2.3 & 4.5 \\
\hline 105 & 2.11 & 4.22 & 2.2 & 4.3 \\
\hline 110 & 1.97 & 3.92 & 2.1 & 4.0 \\
\hline 115 & 1.81 & 3.61 & 1.9 & 3.5 \\
\hline 120 & 1.66 & 3.33 & 1.7 & 3.2 \\
\hline 125 & 1.53 & 3.06 & 1.6 & 3.0 \\
\hline 130 & 1.4 & 2.8 & 1.4 & 2.7 \\
\hline
\end{tabular}

to the configuration of section 4.2.2. The curve in Figure 9 shows the variation trend of the magnetic induction intensity with the increasing of distance. When the coil current keeps the same, the experimental and simulation data are consistent and the magnetic field increases to a certain peak value and then decreases gradually as the distance increases. It can also be seen from Figure 9 that the magnetic induction intensity doubles as the coil current doubles.

\subsubsection{Current control method}

We use the PWM signal to drive the coils; the relationship between the PWM duty ratio and the coil current is shown in Figure 10, where the horizontal coordinate denotes the PWM duty ratio, and the vertical coordinate denotes the coil current. As shown in Figure 10, the current and duty ratio have an approximately linear relationship. However, the current increases circuitously when it is over $2000 \mathrm{~mA}$. This is because the coil temperature gradually increases, and the coil resistance increases as well. Therefore, the coil current decreases, and its change is not obvious as the PWM duty ratio increases. It is worth noting that a current over $2000 \mathrm{~mA}$ is acquired in several periods of time (power off, waiting for the coils to cool).

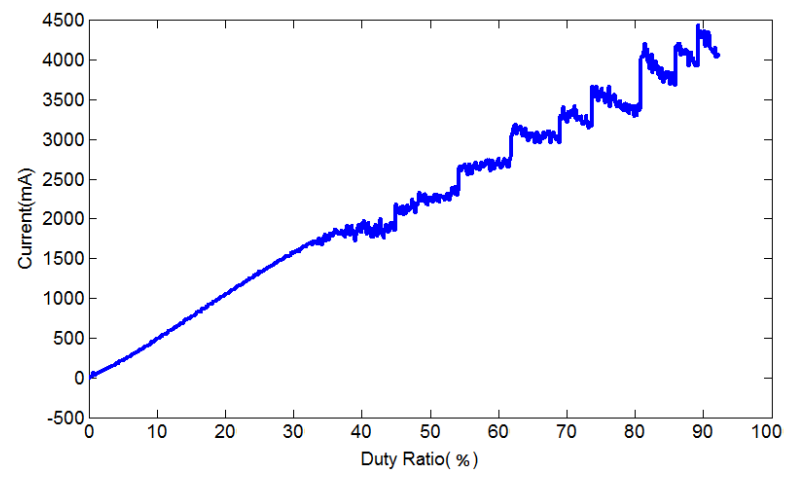

Figure 10: Relationship between the PWM duty ratio and the coil current.

Figure 10 shows that the PWM duty ratio and coil current do not have a linear relationship, which will lead considerable error when using the PWM signal to directly drive the coils, and then affect the haptic feedback. We propose a self-adaptive fuzzy PID algorithm to intelligently control the coil current. To evaluate the performance of the proposed method, we compare our method with the classical PID algorithm. In the experiment, we adjust different currents using these two methods (The current range is $300-3000 \mathrm{~mA}$, and the step is $300 \mathrm{~mA}$ ). For each current, we analyze the maximum error and the standard deviation of 1000 adjusted samples. The comparison of our method and the classical PID algorithm are shown in Figure 11. The blue line and red line are the maximum error and the standard deviation of our method, respectively. It can be observed that the maximum error and the standard deviation of our method are relatively small, which helps to ensure the stability of the current control. The parameters of the classical PID algorithm are $K_{p}=0.2, K_{i}=0.5$, and $K_{d}=0.1$, and these are the best parameters when the current is $1500 \mathrm{~mA}$ for the classical PID algorithm. In our intelligent control method, the parameters above are used as the initial parameters.

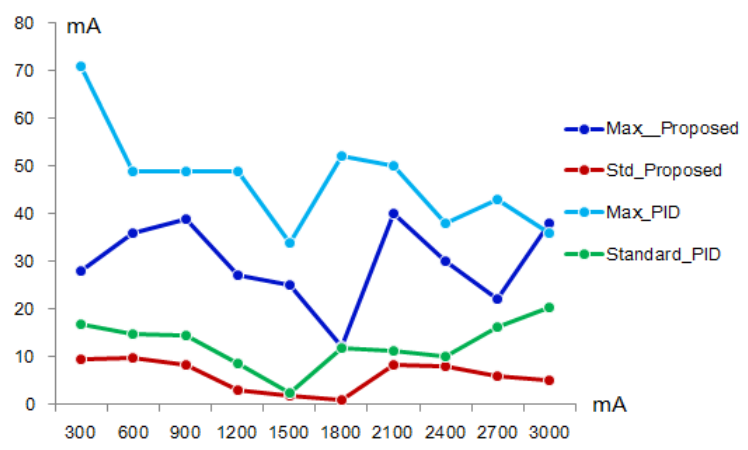

Figure 11: Comparison between our current control method and the classical PID algorithm.

\subsection{Qualitative experiment}

To evaluate the performance of our magnetic levitation haptic device on augmenting the perception of tissue stiffness, we have conducted two qualitative experiments. Experiment 1 is used to evaluate the reliability of our haptic device to distinguish objects of different stiffness. Experiment 2 is used to evaluate the accuracy rate of detecting tissue abnormality. The goal of experiment 2 is to assess whether the designed device has the potential for palpation simulation or not.

\subsubsection{Participants}

We recruited 22 participants ( 9 females and 13 males; with a mean age of 28 years old) among students and teachers. To ensure the objectivity of the experiments, nineteen participants had no previous experience with our magnetic haptic device. All of the participants did not know the goal and design of the experimental.

\subsubsection{Apparatus}

The test hardware is our magnetic levitation haptic prototype device described in section 3 . Three coils of the device are placed $120^{\circ}$ apart. The angle between each coil and the horizontal plane is $60^{\circ}$, and the distance between two coils is $40 \mathrm{~mm}$. The coil attitude is determined through many experiments, and the power consummation of our haptic device is lower during the whole experiment if placing the three coils according to the attitude above.

The Phantom Omni is used to compare with our device for the perception of the tissue stiffness in the qualitative experiment. It is the most cost-effective haptic device available today.

The tasks of calculating our simulation model, solving the coil current and tracking the magnetic stylus are performed using a PC (Intel Xeon CPU E3-1230 V2 @ 3.30 GHz, 8.00 GB of memory). 
This configuration can fulfill the real-time haptic interaction of our experiments. However, if the simulation model is more complex or the number of calculations is larger, more than one computing device may be required to complete the computing tasks.

\subsubsection{Design}

To test the availability of our haptic device for augmenting the perception of tissue stiffness, we devised experiment 1: the kidney model. We designed six levels of stiffness for the kidney model from small to large: A B, C, D, E, and F. The appearances of all six objects are identical. Objects A, C, and E are first used to familiarize the participants with our haptic device and to train them to experience the process of tissue stiffness perception. Next, we disrupt the orders of $\mathrm{A}, \mathrm{C}, \mathrm{E}$ and $\mathrm{B}, \mathrm{D}, \mathrm{F}$ respectively, and the participants are asked to sort the objects according to their perception experience by using the designed haptic device and the Phantom Omni. Figure 12 is a schematic view, which indicates that the deformation varies when exerted the same force on tissues of different stiffness.
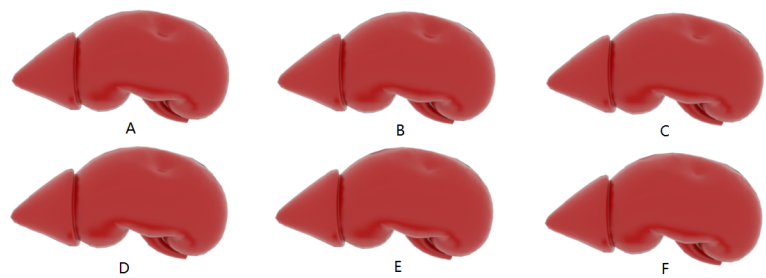

Figure 12: Schematic view of kidney models of different stiffness. Their deformation varies when exerted the same force.

We designed experiment 2 to evaluate the performance of our haptic device for detecting tissue abnormality on two kidney models. We embedded a lump in a kidney as a tissue abnormality, and the other kidney is a normal tissue. The participants are asked to "touch" the kidneys in the virtual environment using our device and the Phantom Omni device and to describe their perception experience.

\subsubsection{Models}

Real-time haptic rendering is very important in the applications of haptic interaction. We model the real-time deformation of kidney using Vega, which is a nonlinear FEM deformable object simulator exploited by Sin et al. [Sin et al. 2013].

The main idea of Vega to realize a rapid deformation model is that the approach exploits dimensional model reduction to build reduced coordinate deformable models for objects with complex geometry [Barbič and James 2005; Barbic 2007]. The motion equation of a deformable solid is described by the Euler-Lagrange equation:

$$
\boldsymbol{M} \ddot{\boldsymbol{u}}+D(\boldsymbol{u}, \dot{\boldsymbol{u}})+R(\boldsymbol{u})=\boldsymbol{f}
$$

where $\boldsymbol{u} \in R^{3 n}$ is the displacement vector (the unknown), $M \in$ $R^{3 n, 3 n}$ is the mass matrix, $D(\boldsymbol{u}, \dot{\boldsymbol{u}})$ are damping forces, and $R(\boldsymbol{u})$ are internal deformation forces. By inserting $\boldsymbol{u}=U \boldsymbol{q}$ into Equation 1 and pre-multiplying by $U^{T}$, they obtain the reduced equations of motion. These equations determine the dynamics of the reduced coordinates $\boldsymbol{q}=\boldsymbol{q}(t) \in R^{r}$ and thus also the dynamics of $\boldsymbol{u}(t)=$ $U \boldsymbol{q}(t)$ :

$$
\ddot{\boldsymbol{q}}+\widetilde{D}(\boldsymbol{q}, \dot{\boldsymbol{q}})+\widetilde{R}(\boldsymbol{q})=\tilde{\boldsymbol{f}}
$$

where $\widetilde{D}, \widetilde{R}, \widetilde{f}$ are $r$-dimensional reduced forces. Similarly, the tangent stiffness matrix can be reduced as the following:

$$
\widetilde{K}(\boldsymbol{q})=U^{T} K(U \boldsymbol{q}) \quad U \in R^{r, r}
$$

We adopt the method above to build the kidney models, and the parameters are as follows: the number of vertices is 16680; the number of elements is 8466 ; the simulation frame rate is 60 . We achieve the real-time deformation simulation for the experiments of real-time tissue stiffness perception.

\subsubsection{Procedure}

Throughout the experiments, the participants individually entered the laboratory, and they had no communication with each other. The two experiments are divided into two stages. After all participants completed the first experiment, we counted and analyzed the experimental results. If the accuracy rate of the participants in distinguishing the tissue stiffness exceeded $80 \%$, we continued to conduct the second experiment.

The participant read and signed an informed consent sheet after entering the laboratory. It is widely known that there may be sickness phenomenon in the experience of AR and VR. We told each participant he or she could interrupt the experiment at any time if encountering problems.

Participants attempted to use our device according to our guidelines. After the preparatory work was completed, the participant conducted the training task. During the training task, objects A, C, and $\mathrm{E}$ were sorted from small to large according to the stiffness. The goal of the training task is to let the participant experience objects of different stiffness.

We conducted the first test of experiment 1 after the training task was completed. The participant was asked to sort the objects A, C, and $\mathrm{E}$, whose orders were shuffled. We then counted the results. The participant was totally unaware of the experimental results.

Then we conducted the second test of experiment 1 . The participant perceived the objects $\mathrm{B}, \mathrm{D}$, and $\mathrm{F}$ and sorted them, and the results were counted. The participant had no interaction with objects B, D, and $\mathrm{F}$ using our haptic device before the test, which helped ensure the credibility of our experiment. The participant would then rest and wait in a separate waiting area after finishing experiment 1.

In experiment 2, each Participant was told to detect whether the kidney model contained lesions in the virtual environment. All participants were aware that the stiffness of diseased tissue is higher than that of normal tissue. As shown in Figure 13, the model contains two kidneys, only one of which is diseased. However, we told the participants that each kidney may be in one of three conditions: (1) Neither contains tissue abnormality; (2) Only one contains tissue abnormality; (3) Both contain tissue abnormality. Each participant conducted experiment 2 directly on the basis of experiment 1. We recorded participant's of the experience on the perception of the two kidneys.
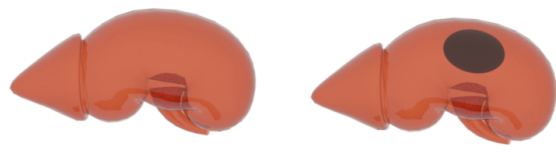

Figure 13: Kidney model. The left is normal and the right is embedded with a lump.

The participants were asked to conduct the experiments above by using our haptic device as well as the Phantom Omni. The experimental data were for comparison. 
Table 3: QoE Questionnaire. Each question is rated on a 5-point scale from 1 (Not at all) to 5 (Totally).

\begin{tabular}{|l|l|}
\hline Factor & Question \\
\hline \multirow{3}{*}{ Sensory } & \begin{tabular}{l} 
How much did the haptic device contribute to \\
augmenting the stiffness perception of tissue? \\
\cline { 2 - 2 }
\end{tabular} \\
$\begin{array}{l}\text { Whether the interaction way is consistent with } \\
\text { your habits of the real world? }\end{array}$ \\
\hline Realism & $\begin{array}{l}\text { How much did the perceived experiences seem } \\
\text { consistent with your real-world experiences? }\end{array}$ \\
\hline Comfort & How comfortable was the haptic device? \\
\hline Satisfaction & How much did you prefer to use the device? \\
\hline
\end{tabular}

\subsubsection{Measurement of QoE: questionnaire}

After finishing experiment 2, each participant was asked to fill in a questionnaire. The questionnaire is designed to evaluate the user's perceived quality of experience (QoE) of the designed haptic device for tissue stiffness perception. QoE is related to the subjective user experience with a service or an application [Kilkki 2008]. The questionnaire is based on four factors, which are similar to the literature [Danieau et al. 2012a; Danieau et al. 2012b]: sensory, realism, comfort and satisfaction. "Sensory" characterizes how much the haptic device contributed to augmenting the stiffness perception of tissue and whether the interaction way is consistent with user's habits of the real world. "Realism" describes how much the virtual environment is realistic. The factor is closely related to the simulation model. "Comfort" measures how comfortable the haptic device is to use. "Satisfaction" determines whether the user prefers to use the device. Each factor was evaluated by questions rated on a 5point scale. A mean was calculated for each factor, and the QoE was computed as the sum of these 4 factors. Table 3 presents the questions used to evaluate the QoE.

\subsubsection{Results}

In Experiment 1, the participants distinguished the kidney models of different stiffness. The statistical results of test 1 and test 2 using our device and Phantom Omni are shown in Figure 14. 21 participants $(95 \%)$ successfully distinguished the objects of different stiffness in test 1 . In test 2 , two participants wrongly sorted the two models of smaller stiffness (objects B and D) using the designed haptic device and two participants appeared the same mistake using Phantom Omni. What is worth mentioning is that the same participant made mistakes in the two tests, and it may result from his poor ability of tactile perception. The accuracy rate of test 2 is $91 \%$, showing that our haptic device has a ability to distinguish objects of different stiffness.

In Experiment 2, the participants detected tissue abnormality. The simulation model contains two kidneys, one is abnormal and the other is a normal tissue. All participants correctly detected the normal tissue and the abnormal tissue by using the two haptic devices.

In addition, a score for the four factors - sensory, realism, comfort and satisfaction - were obtained using a questionnaire. The QoE is the sum of the four factors. The statistical results are shown in Figure 15 and Table 4 . In Table 4, we counted the means $\bar{x}$ and standard deviations $\sigma_{x}$ of each factor score. In addition, we conducted a nonparametric test to analyze the statistical results. The QoE of our haptic device is not significantly different from that of Phantom Omni $\left(Q o E_{\text {Our }}=15.09 \approx Q o E_{\text {Phantom }}=15.55\right.$, MannWhitney U test $p=0.1573>0.05$, effect size is $E S=0.52$ and statistical power is power $=0.50$ ). Therefore, our device significantly enhances the quality of experience. The "Sensory" score of our device is higher than Phantom Omni. Taking into account

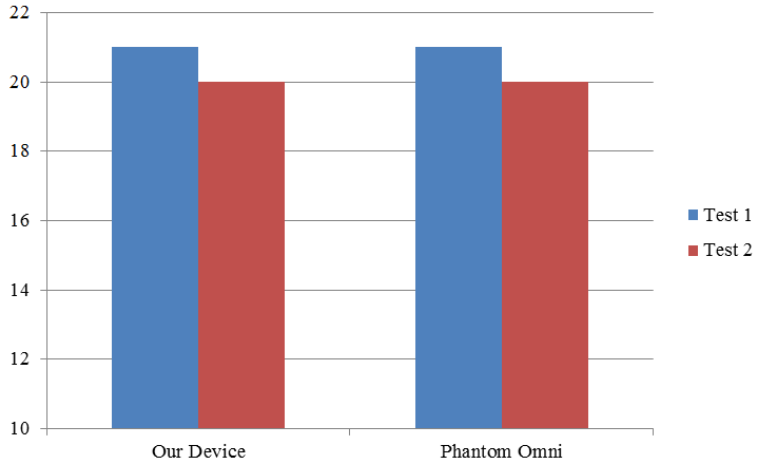

Figure 14: Number of participants to accurately distinguish corresponding objects using the designed device and Phantom Omni.

that the accuracy rate of distinguishing objects of different stiffness is identical using the two devices, the higher score of "Sensory" is possible due to the reason that the interaction way of our device is closer to the natural manner.

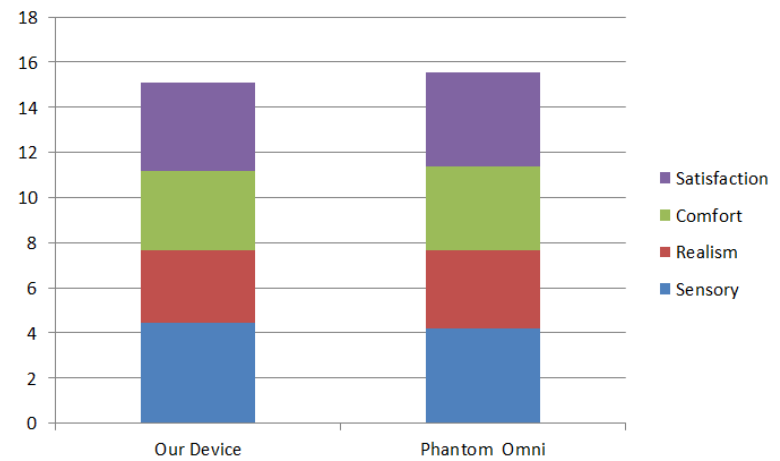

Figure 15: Quality of experience. The QoE of our haptic device is not significantly different from that of Phantom Omni.

\section{Discussion}

The quantitative results show that the simulation data of the magnetic induction intensity used to generate force feedback is consistent with the experimental data, and our current control algorithm is accurate, which ensures the performance of the designed haptic device. The preliminary qualitative results demonstrate that our device can help users to distinguish objects of different stiffness and correctly detect tissue abnormality in the virtual environment. Therefore, the designed device has the potential for medical training and RMIS to augment the perception of tissue.

Although the QoE of our haptic device is lower than that of Phantom Omni, the "Sensor" score of our device is higher. Our device does not require mechanical transmission and the magnetic stylus is flexible, thus the user can interact with virtual objects in a natural manner. Therefore, the higher Sensor score is benefit from the question "Whether the interaction way is consistent with your habits of the real world". As we all know surgeons use their fingers to conduct physical examination in real palpation. A magnetic glove or a magnetic ring may give surgeons a more favorable experience, which can enable them to perceive the objects in the virtual environment in a natural manner.

In this paper, tissue models were built by using the Vega software, 
Table 4: Means $(\bar{x})$ and standard deviations $\left(\sigma_{x}\right)$ for each device with respects to each factor.

\begin{tabular}{|l|l|l|l|l|l|l|}
\hline Factor & QoE & Sensory & Realism & Comfort & Satisfaction & \\
\hline \multirow{2}{*}{ Our Device } & 15.09 & 4.45 & 3.23 & 3.50 & 3.91 & $\bar{x}$ \\
\cline { 2 - 7 } & 0.9715 & 0.5096 & 0.5284 & 0.5118 & 0.2942 & $\sigma_{x}$ \\
\hline \multirow{2}{*}{ Phantom Omni } & 15.55 & 4.19 & 3.45 & 3.73 & 4.18 & $\bar{x}$ \\
\cline { 2 - 7 } & 0.8004 & 0.3948 & 0.5096 & 0.5505 & 0.3948 & $\sigma_{x}$ \\
\hline
\end{tabular}

which achieves real-time haptic interactive simulation by reducing the dimension of the model. However, the process of dimension reduction would affect the accuracy of the force feedback, which is one reason why the score of "Realism" factor is slightly lower in the questionnaire. In addition, we simulate tissues of different stiffness by setting different stiffness matrix for the objects and we obtained a tissue abnormality by embedding a lump into a normal tissue, which also affects the realistic of our model. In the qualitative experiment, two participants wrongly distinguish objects B and $\mathrm{D}$, which may due to the model. A high precision simulation model that is for the real-time haptic interaction needs to be studied to achieve realistic perception of tissue stiffness.

For the design of our haptic device, we have reasonably explained the design of the coil array compared to the existing methods. However, we just find a relatively suitable coil attitude using the experimental method, which may not be the best attitude. To obtain the best coil attitude conveniently, we need to describe the interactive requirements and design a solving model. In addition, the "Comfort" score of our device is low and some participants reflected that the movement of the magnetic stylus is slightly different from that of the virtual stylus during interactions with the virtual objects using our device. The synchronization of visual and tactile may cause the questions above.

Despite our device still has some limitations, the preliminary experiments confirm that the prototype device can be used to enhance the perception of objects in virtual environment. We will improve the device according to user feedback comments and the present defects.

\section{Conclusions}

We devise a magnetic levitation haptic device to augment the stiffness perception of tissue. The device eliminates mechanical friction, which helps to improve the accuracy of users' perception. The operator can sense the tissue in the virtual environment using the magnetic stylus to detect the presence of tissue lesions in a natural manner. We conduct a simulation analysis of the EMF generated by the designed adjustable coil array. We provide a reasonable explanation of the placement method of the coil array compared with the existing methods, and we adjust the coils just via the experimental method in this paper. In the experiments, the participants can accurately distinguish objects of different stiffness, as well as precisely detect the tissue abnormality using our haptic device, and the accuracy rate of detecting tissue abnormality is also very high.

In future work, we will build a model to describe the interaction requirement and design a solving model of the most suitable attitude for the coils. If the best coil attitude can be solved out according to different interaction requirements, our haptic device will be more easily expanded to other applications. We will try to design a magnetic glove or a magnetic ring to directly fulfill the application of touching virtual objects. In addition, we will build a more realistic abnormal tissue by acquiring the parameters of the real animal tissue and invite experts to evaluate the improved haptic device in our future work.

\section{Acknowledgements}

We thank all the volunteers. This work was supported by the National Natural Science Foundation of China under Grant No.61373107 and Wuhan Science and Technology Bureau under Grant No. 2016010101010022.

\section{References}

Arafsha, F., Zhang, L., Dong, H., And El Saddik, A. 2015. Contactless haptic feedback: state of the art. In Haptic, Audio and Visual Environments and Games (HAVE), 2015 IEEE International Symposium on, IEEE, 1-6.

BARBIČ, J., AND JAMES, D. L. 2005. Real-time subspace integration for st. venant-kirchhoff deformable models. In $A C M$ transactions on graphics (TOG), vol. 24, ACM, 982-990.

BARBIC, J. 2007. Real-time reduced large-deformation models and distributed contact for computer graphics and haptics. $\mathrm{PhD}$ thesis, Intel.

Berkelman, P., AND Dzadovsky, M. 2008. Magnet levitation and trajectory following motion control using a planar array of cylindrical coils. In ASME 2008 Dynamic Systems and Control Conference, American Society of Mechanical Engineers, 923930.

Berkelman, P. J., AND DZAdovsky, M. 2009. Large motion range magnet levitation using a planar array of coils. In ICRA, $1609-1610$.

Berkelman, P., ANd Dzadovsky, M. 2013. Magnetic levitation over large translation and rotation ranges in all directions. IEEE/ASME Transactions on Mechatronics 18, 1, 44-52.

Berkelman, P., AND Hollis, R. L. 1997. Dynamic performance of a magnetic levitation haptic device. In Intelligent Systems \& Advanced Manufacturing, International Society for Optics and Photonics, 140-149.

Berkelman, P. J., AND Hollis, R. L. 2000. Lorentz magnetic levitation for haptic interaction: Device design, performance, and integration with physical simulations. The International Journal of Robotics Research 19, 7, 644-667.

Berkelman, P., Miyasaka, M., And Anderson, J. 2012. Co-located 3d graphic and haptic display using electromagnetic levitation. In 2012 IEEE Haptics Symposium (HAPTICS), IEEE, 77-81.

Berkelman, P., Bozlee, S., AND Miyasaka, M. 2013. Interactive dynamic simulations with co-located maglev haptic and $3 \mathrm{~d}$ graphic display. In Int. Conf. Advances in Computer-Human Interactions, Citeseer, 324-329.

Berkelman, P. 2007. A novel coil configuration to extend the motion range of lorentz force magnetic levitation devices for haptic interaction. In 2007 IEEE/RSJ International Conference on Intelligent Robots and Systems, IEEE, 2107-2112. 
Bethea, B. T., Okamura, A. M., Kitagawa, M., Fitton, T. P., Cattaneo, S. M., Gott, V. L., Baumgartner, W. A., AND YUH, D. D. 2004. Application of haptic feedback to robotic surgery. Journal of Laparoendoscopic \& Advanced Surgical Techniques 14, 3, 191-195.

Danieau, F., Fleureau, J., Cabec, A., Kerbiriou, P., GuilLotel, P., Mollet, N., Christie, M., And LÉCuyer, A. 2012. Framework for enhancing video viewing experience with haptic effects of motion. In 2012 IEEE Haptics Symposium (HAPTICS), IEEE, 541-546.

Danieau, F., Fleureau, J., Guillotel, P., Mollet, N., LÉCUYER, A., AND Christie, M. 2012. Hapseat: producing motion sensation with multiple force-feedback devices embedded in a seat. In Proceedings of the 18th ACM symposium on Virtual reality software and technology, ACM, 69-76.

De Gersem, G., VAn Brussel, H., And Tendick, F. 2005. Reliable and enhanced stiffness perception in soft-tissue telemanipulation. The international journal of robotics research 24, 10 , 805-822.

Gwilliam, J. C., Pezzementi, Z., Jantho, E., Okamura, A. M., AND HsiaO, S. 2010. Human vs. robotic tactile sensing: Detecting lumps in soft tissue. In 2010 IEEE Haptics Symposium, IEEE, 21-28.

Hollis, R. L., Salcudean, S. E., And Allan, A. P. 1991. A six-degree-of-freedom magnetically levitated variable compliance fine-motion wrist: design, modeling, and control. IEEE Transactions on Robotics and Automation 7, 3, 320-332.

Hollis JR, R. L., AND SAlCUdeAn, S. E., 1992. Input/output system for computer user interface using magnetic levitation, Sept. 8. US Patent 5,146,566.

Horeman, T., Rodrigues, S. P., VAn den Dobbelsteen, J. J., Jansen, F.-W., And DAnkelman, J. 2012. Visual force feedback in laparoscopic training. Surgical endoscopy 26, $1,242-248$.

Hoshi, T., Abe, D., And Shinoda, H. 2009. Adding tactile reaction to hologram. In $R O-M A N$, Citeseer, 7-11.

Hoshi, T. 2011. Development of aerial-input and aerial-tactilefeedback system. In World Haptics Conference (WHC), 2011 IEEE, IEEE, 569-573.

Hu, J., Chang, C., Tardella, N., Pratt, J., English, J., ET AL. 2006. Effectiveness of haptic feedback in open surgery simulation and training systems. Stud. Health Technol. Inform $119,213-218$.

HU, J., 2005. Magnetic haptic feedback systems and methods for virtual reality environments, June 1. US Patent App. 11/141,828.

Kesner, S. B., And Howe, R. D. 2011. Discriminating tissue stiffness with a haptic catheter: Feeling the inside of the beating heart. In World Haptics Conference (WHC), 2011 IEEE, IEEE, $13-18$.

KILKKI, K. 2008. Quality of experience in communications ecosystem. J. UCS 14, 5, 615-624.

Li, M., LiU, H., Li, J., Seneviratne, L. D., And Althoefer, K. 2012. Tissue stiffness simulation and abnormality localization using pseudo-haptic feedback. In Robotics and Automation (ICRA), 2012 IEEE International Conference on, IEEE, 53595364.
Li, M., Faragasso, A., Konstantinova, J., Aminzadeh, V., Seneviratne, L. D., Dasgupta, P., And Althoefer, K. 2014. A novel tumor localization method using haptic palpation based on soft tissue probing data. In 2014 IEEE International Conference on Robotics and Automation (ICRA), IEEE, $4188-4193$.

Liu, H., Noonan, D. P., Althoefer, K., and Seneviratne, L. D. 2008. Rolling mechanical imaging: a novel approach for soft tissue modelling and identification during minimally invasive surgery. In Robotics and Automation, 2008. ICRA 2008. IEEE International Conference on, IEEE, 845-850.

Liu, H., Li, J., Poon, Q.-I., Seneviratne, L. D., And AlTHOEFER, K. 2010. Miniaturized force-indentation depth sensor for tissue abnormality identification during laparoscopic surgery. In Robotics and Automation (ICRA), 2010 IEEE International Conference on, IEEE, 3654-3659.

Liu, H., Li, J., Song, X., Seneviratne, L. D., And AlthoeFER, K. 2011. Rolling indentation probe for tissue abnormality identification during minimally invasive surgery. IEEE Transactions on Robotics 27, 3, 450-460.

Long, B., Seah, S. A., Carter, T., and Subramanian, S. 2014. Rendering volumetric haptic shapes in mid-air using ultrasound. ACM Transactions on Graphics (TOG) 33, 6, 181.

Nisky, I., Huang, F., Milstein, A., Pugh, C. M., MussaIVALDI, F. A., AND KARNIEL, A. 2012. Perception of stiffness in laparoscopy-the fulcrum effect. Studies in health technology and informatics 173,313 .

QueK, Z. F., Schorr, S. B., Nisky, I., OKamura, A. M., AND PROVANCHER, W. R. 2014. Augmentation of stiffness perception with a 1-degree-of-freedom skin stretch device. IEEE Transactions on Human-Machine Systems 44, 6, 731-742.

RoвотіCs, F. M. 1993. Lorentz levitation technology: a new approach to fine motion robotics, teleoperation, haptic interfaces, and vibration isolation $\mathrm{rl}$ hollis se salcudean. Proc. Fifth Intl Symp. Robotics Research, 1-18.

Sin, F. S., Schroeder, D., ANd BARbič, J. 2013. Vega: Nonlinear fem deformable object simulator. In Computer Graphics Forum, vol. 32, Wiley Online Library, 36-48.

Sodhi, R., Poupyrev, I., Glisson, M., AND Israr, A. 2013. Aireal: interactive tactile experiences in free air. ACM Transactions on Graphics (TOG) 32, 4, 134.

Son, H. I., Bhattacharjee, T., And Hashimoto, H. 2012. Effect of impedance-shaping on perception of soft tissues in macro-micro teleoperation. IEEE Transactions on Industrial Electronics 59, 8, 3273-3285.

Song, A., Morris, D., Colgate, J. E., And Peshinin, M. A. 2005. Real time stiffness display interface device for perception of virtual soft object. In 2005 IEEE/RSJ International Conference on Intelligent Robots and Systems, IEEE, 139-143.

Stetten, G., Wu, B., Klatzky, R., Galeotti, J., Siegel, M., Lee, R., Mah, F., Eller, A., Schuman, J., AND HolLIS, R. 2011. Hand-held force magnifier for surgical instruments. In International Conference on Information Processing in Computer-Assisted Interventions, Springer, 90-100.

Wu, B., Klatzky, R. L., AND Hollis, R. L. 2011. Force, torque, and stiffness: Interactions in perceptual discrimination. IEEE transactions on haptics 4, 3, 221-228. 